\title{
Long-term evolution of small icy bodies of the Solar System
}

\author{
Dina Prialnik \\ Dept. of Geophysics \& Planetary Sciences, Tel Aviv University \\ Ramat Aviv, Tel Aviv 69978, Israel \\ email: dina@planet.tau.ac.il
}

\begin{abstract}
Detailed evolutionary calculations spanning $4.6 \times 10^{9}$ yr are presented for (a) a model representing main-belt comet 133P/Elst-Pizarro, considering different initial mixtures of ices and dust, and (b) a Kuiper Belt object heated by radioactive decay, growing in size from an initial radius of $10 \mathrm{~km}$ to a final $250 \mathrm{~km}$.

It is shown that for the main-belt comet only crystalline $\mathrm{H}_{2} \mathrm{O}$ ice may survive in the interior of the nucleus, and may be found at depths ranging from $\sim 50$ to $150 \mathrm{~m}$. Other volatiles will be completely lost. For the large Kuiper Belt object, evaporation and flow of water and vapor gradually remove the water from the core and the final (present) structure is differentiated, with a rocky, highly porous core of $80 \mathrm{~km}$ radius. Outside the core, due to refreezing of water vapor, a compact, ice-rich layer forms, a few tens of $\mathrm{km}$ thick. The amorphous ice is preserved in an outer layer about $20 \mathrm{~km}$ thick.
\end{abstract}

Keywords. comets: general, comets: individual (133p/Elst-Pizarro), Kuiper Belt

\section{Introduction}

A new class of comets has been identified in recent years - known as "main-belt comets" (MBCs) - the prototype being 133P/Elst-Pizarro (EP), which was first classified as a main-belt asteroid. Cometary activity for EP was first detected in 1996 as a dust tail, and at the time it was thought to be the result of an impact. The dust could be either a trail of debris (Toth 2000), or the manifestation of volatile-driven activity caused by the exposure of deep-lying ice (Boehnhardt et al. 1998). The latter interpretation gained support when the comet became active again one orbital period later, in 2002 (Hsieh et al. 2004, Ferrin 2006). The third episode of activity detected near perihelion another orbital period later (Jewitt et al. 2007b) left little doubt that mass ejection was triggered by volatile sublimation. Two more objects belonging to the MBC class soon joined EP: 176P/LINEAR and P/2005 U1 (Read), which showed vigorous activity (Hsieh et al. 2009a,b) and recently, a fourth object was detected, P/2008 R1 (Jewitt et al. 2009).

The orbits of these objects are stable; hypotheses regarding their origin are divided between formation in place (Haghighipour 2008) and capture from distant regions of the Solar System into the outer asteroid belt during the late heavy-bombardment era (Levison et al. 2008, based on the Nice model). Either way, MBCs must have spent between 3.9 and 4.6 Byr in the main belt. A plausible scenario that would explain both the prolonged nature of the dust emission and its recurrence at successive perihelia is sublimation of ices exposed by a recent impact, which implies that MBCs have ices buried below the surface. The question whether or not may ices survive 4.6 Byr of evolution in an orbit that is relatively close to the Sun requires detailed evolutionary calculations. Simplified analytical estimates (Schorghofer 2008) indicate that this may be possible, but 
such estimates do not consider gas flow through the porous nucleus, nor sublimation in the deep porous interior.

A different question that demands long-term evolutionary calculations is whether and to what extent are comets, or rather, their distant progenitors - Kuiper Belt objects (KBOs) - pristine bodies that hold clues to the formation of the solar system. Simplified models show that radiogenic heating by short-lived radionuclides, such as ${ }^{26} \mathrm{Al}$, provides sufficient energy for melting the ice (e.g., Merk and Prialnik 2006, Podolak and Prialnik 2006, Jewitt et al. 2007a and McKinnon et al. 2007).

In this paper long-term evolutionary calculations for a MBC (Prialnik \& Rosenberg 2009) and a KBO (Prialnik \& Merk 2008) - meant to shed light on these questions - are presented and compared.

\section{Long-term evolution - 4.6 Byr - of a Main-Belt comet}

The code used for numerical modeling is a 1-D code described in detail by Prialnik (1992) and Sarid et al. (2005); discussion of the input physics may be found in Prialnik et al. (2004). The orbital period of EP is $\sim 5.5 \mathrm{yr}$ and in the long-term calculations aim to cover 4.6 Byr of evolution. Clearly, orbital changes cannot be resolved, as this would require a prohibitive number of time steps. To circumvent this difficulty, but still keep track of the correct overall amount of energy absorbed by the comet, the elliptic orbit is replaced by a circular one, corresponding to the same total orbital insolation. Different initial configurations are considered, having in common a radius of $2.5 \mathrm{~km}$, an albedo of 0.04 , and dust and ice in equal mass fractions.

For the first model (Model A), the initial composition is a mixture of pure amorphous water ice (no occluded gases) and dust, representing a captured body, formed in more distant and cold regions of the Solar System. The ice crystallizes completely during the first $6 \times 10^{5}$ yr of evolution, with the crystallization front - initiated by absorption of solar energy - advancing from the surface all the way to the center, feeding mainly on the released latent heat. If gases were trapped in the amorphous ice, they would have escaped to the surface and out of the nucleus. While the crystallization front is receding toward the center, the crystalline ice starts sublimating at the surface, with the sublimation front very slowly advancing inwards, leaving behind an ice-depleted dust mantle. Some of the vapor flows inwards and refreezes ahead of the crystallization front. Thus the ice fraction profile peaks at some depth below the dust mantle, as shown in Fig. 1. This peak is also fed by vapor flowing outward from the deep interior, where the temperature has risen to about $125 \mathrm{~K}$, allowing for bulk sublimation. Hence the ice is slowly depleted, but far more slowly than the rate of free sublimation would imply, impeded by the build-up of internal pressure. As a result of internal sublimation, after $4.6 \times 10^{9} \mathrm{yr}$ of evolution, the ice density throughout most of the interior is significantly lower than the initial density, as seen in Fig. 1. Overall, the total ice content of the body at present is slightly less than half of the initial. The depth at which ice may be found at present, assuming no collapse of the dust mantle, is about $90 \mathrm{~m}$.

If the body formed in place in the asteroid belt, it is more likely that the ice was crystalline to start with. A second model is evolved (Model B), identical to the first, except that the ice is initially crystalline throughout. In this case the internal temperature attained is slightly lower, and less ice sublimates both in the interior and below the surface, so that ice may be found at present at a depth of $\sim 45 \mathrm{~m}$ (see Fig. 1). The difference between the amorphous and crystalline ice bodies is due to the additional heat source in the former, provided by the exothermic crystallization.

In order to assess the effect of initial density (and thus ice content), a third model (Model C) is calculated, identical to the second, except that the bulk initial density is 

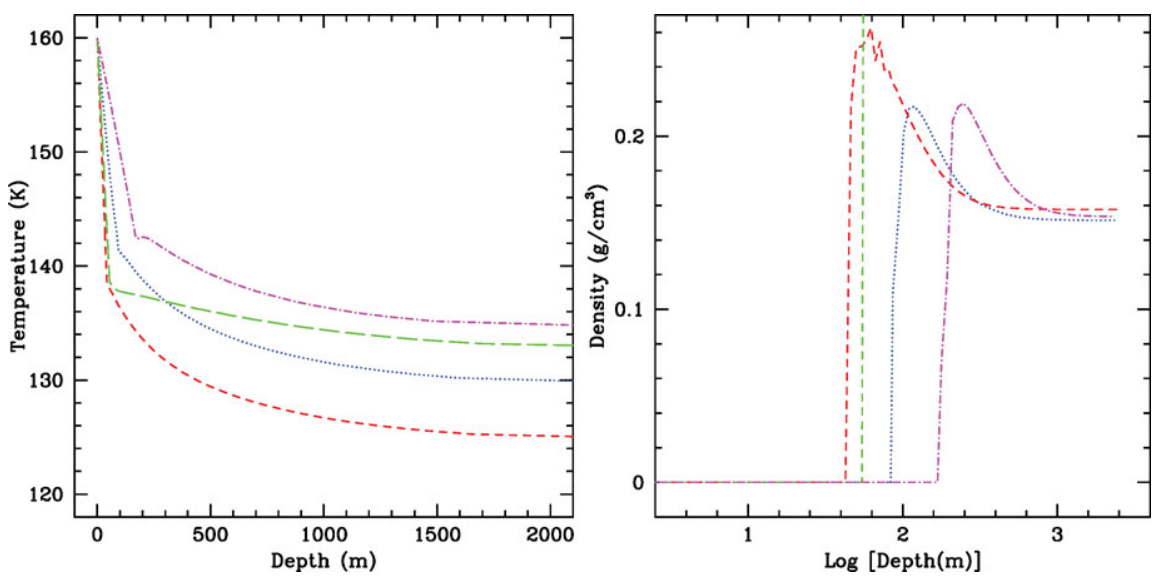

Figure 1. Comparison of the present structure of the 4 models: A (blue dot), B (red short dash), C (green long dash) and D (magenta dash-dot), for temperature profile (left) and $\mathrm{H}_{2} \mathrm{O}$ density (right).

about 2.5 times larger and the porosity - correspondingly lower. This causes the internal vapor pressure to rise and hence the temperatures attained are higher. The relatively high pressure is maintained due to the lower porosity that hinders the flow of gas. In fact, the outflow of vapor is so weak, that only a very small fraction of the ice sublimates in the interior. The $\mathrm{H}_{2} \mathrm{O}$-depleted layer is about $50 \mathrm{~m}$ thick, similar to the previous case, but the internal density of ice remains almost unchanged.

The next model (Model D) includes a mixture of 4 volatile ices - $\mathrm{CO}_{2}, \mathrm{HCN}, \mathrm{NH}_{3}$ and $\mathrm{C}_{2} \mathrm{H}_{2}$ - besides crystalline water ice and dust. Water ice amounts to only 0.45 of the mass, the remaining 0.05 being divided equally among the other volatiles. The significant result is that after about $10^{8} \mathrm{yr}$, no volatile beside $\mathrm{H}_{2} \mathrm{O}$ is left, not even a trace. As for the $\mathrm{H}_{2} \mathrm{O}$ ice, the behavior is similar to the other cases, but sublimation is strongest in this case, with the ice boundary receding down to $\sim 150 \mathrm{~m}$. Over 3 times more $\mathrm{H}_{2} \mathrm{O}$ ice is lost as compared to Model B (of the same density), in addition to the other volatiles. Energetically, this does not pose a problem since the energy required for sublimating the entire ice content of the body is only about $10^{-7}$ of the absorbed solar energy during the lifetime of the solar system.

\section{Long-term evolution - 4.6 Byr - of a Kuiper-Belt object}

The generic KBO considered has a radius of $250 \mathrm{~km}$ and the main energy source in this case is radioactive decay of ${ }^{26} \mathrm{Al}$ and ${ }^{40} \mathrm{~K}$. The evolution starts with a $100 \mathrm{Myr}$-long accretion episode, during which the body grows in size from an initial radius of $10 \mathrm{~km}$ to the final radius, amounting to a volume (mass) increase by a factor of $1.56 \times 10^{4}$. However, since the half lifetime of ${ }^{26} \mathrm{Al}(0.72 \mathrm{Myr})$ is far shorter than the accretion phase, most of the radiogenic heating by this short-lived nuclide occurs while the body has only increased its volume by only a factor of 10 . The central temperature reaches its peak after less than 2 Myr of growth and then gradually declines.

The radioactive energy released by the decay of the longer lived radioisotope ${ }^{40} \mathrm{~K}$ (half lifetime of $1.28 \mathrm{Byr}$ ), is supplied at too low a rate to compete with conductive cooling. Eventually, however, the heat diffusion timescale of the growing body exceeds the radioactive heating timescale, and the central temperature starts rising again, when about one third of the mass has been accreted and the radius has attained $\sim 175 \mathrm{~km}$. It 

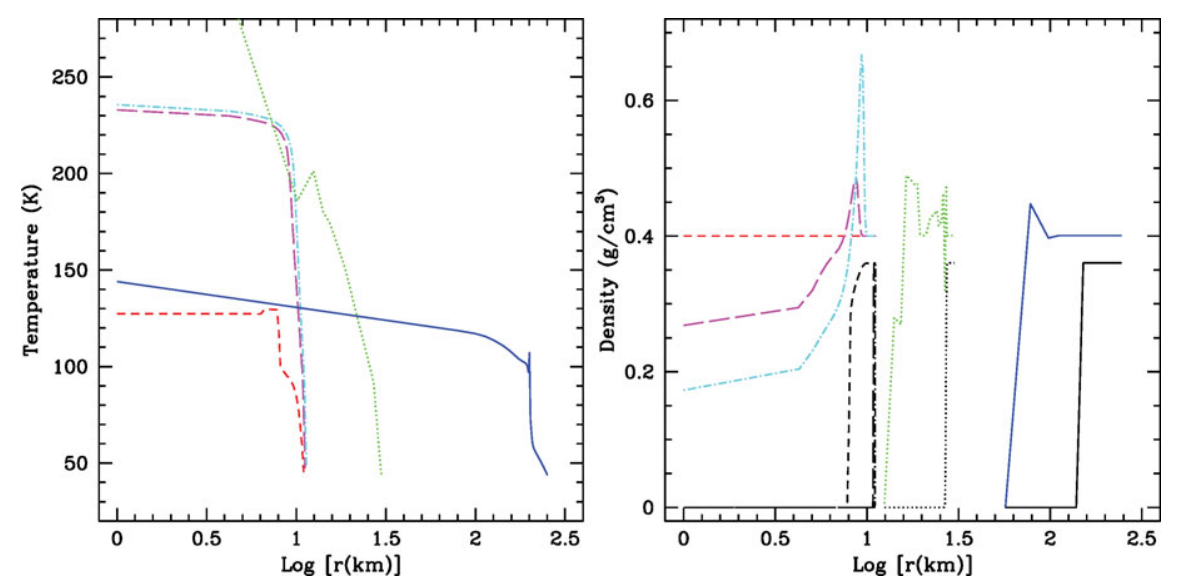

Figure 2. Profiles of temperature (left) and ice density (right) at various times during evolution: early evolution, at times: $1 \times 10^{5}$ yr (short-dashed line, red), $2 \times 10^{5}$ yr (long-dashed line, magenta), $3 \times 10^{5}$ yr (dash-dotted line, cyan), and $4 \times 10^{6} \mathrm{yr}$ (dotted line, green); and present time, $4.6 \times 10^{9}$ yr (solid line, blue). Black (on the right) represents amorphous ice.

reaches another, lower, peak after the body has reached its final size. The decay of ${ }^{40} \mathrm{~K}$ is then followed by a slow decline in central temperature to the present value that still exceeds the equilibrium temperature at the body's average heliocentric distance.

The evolution of the composition is shown in Figure 2 by profiles of the ice density as function of radial distance at different times. When the central temperature - initially $70 \mathrm{~K}$ throughout - exceeds $\sim 120 \mathrm{~K}$, the amorphous ice starts crystallizing and a crystallization front advances from the center outwards. As the temperature rises further, the crystalline ice in the core begins to sublimate and the vapor flows outwards. Upon reaching colder layers, most of it refreezes. Slowly, the core becomes depleted of ice, while further out the ice content increases, as exhibited by the early profiles of Fig. 2 . Under the assumed conditions, this already happens before the melting temperature is attained. A considerable amount of water is produced only when the permeability to flow is significantly reduced. In all cases, however, the core becames eventually ice depleted.

Although by the end of the accretion phase, most of the ice is still amorphous, it slowly crystallizes during the first $1.5 \times 10^{9}$ years of evolution. The heat released by the exothermic transition delays cooling of the body. But at the end of 4.6 Byr, the $\mathrm{KBO}$ is cold, with the temperature ranging from slightly above $60 \mathrm{~K}$ at the center to $\sim 40 \mathrm{~K}$ at the surface (the average equilibrium temperature). The layered structure has a very porous rocky core, $\sim 80 \mathrm{~km}$ in radius (assuming that the material strength is high enough to prevent collapse), completely depleted of ice. Despite the fact that the core radius reaches out to almost $1 / 3$ of the object's size, the core's volume constitutes only $3.3 \%$ of the total volume, and an even smaller fraction of its mass (the core porosity being higher than the average porosity).

Surrounding the core there is a relatively thin layer enriched in ice; its volume is roughly the same as the core's. The higher ice content is a result of refreezing of the water and vapor flowing out of the hot core into the cooler outer layers during the early stages of evolution. The bulk of the configuration has ice and dust in roughly the initial proportions, but the initially amorphous ice is now crystallized. Surrounding it is a thin layer, of amorphous and crystalline ice, with the ratio between them gradually increasing outwards. Finally, the surface $\sim 20 \mathrm{~km}$ thick layer, comprising $22 \%$ of the volume, preserves the initial composition. 


\section{Conclusions}

In conclusion, $\mathrm{H}_{2} \mathrm{O}$ ice may be retained in the interior of a main belt body, despite its proximity to the sun and the long evolution time in its orbit. The ice is expected to be found at a depth of $\sim 50-150 \mathrm{~m}$, depending on initial structure and composition, but no other volatile ices would be able to survive. Therefore, any activity resulting from exposure of the ice-rich layer by an impact, will be driven by water sublimation and should be compatible with the $\mathrm{H}_{2} \mathrm{O}$ sublimation pattern. This does not exclude, however, the presence of other volatile species, which may remain trapped in a fraction of the amorphous ice that does not crystallize, as indicated by laboratory experiments (Notesco \& Bar-Nun 1997). These volatiles will be ejected together with the water molecules.

Regarding KBOs, pristine material may be retained in a relatively thick outer layer, out of which comets may be broken off by collisions (Davis \& Farinella 1997). Beneath this layer, the ice is crystallized, while a central core is porous and completely ice-depleted. This layered structure is the typical outcome of long-term evolution under the initial conditions assumed, while changes in physical parameters merely affect to some extent the relative thicknesses of the various layers.

Finally, it is interesting to note that the two types of icy bodies considered here, although very different in nature, location in the Solar System, and major heat source, have in common an ice-depleted zone that amounts to no more than $10 \%$ of ther volume, and - at the boundary of this zone - an ice-enriched layer resulting from vapor migration and refreezing.

\section{References}

Boehnhardt, H., Sekanina, Z., Fiedler, A., Rauer, H., Schulz, R., \& Tozzi, G. 1998, Highlights of Astronomy, 11, 233

Davis, D. R. \& Farinella, P. 1997, Icarus, 125, 50

Ferrín, I. 2006, Icarus, 185, 523

Haghighipour, N. 2008, LPI, 1405, 8287

Hsieh, H. H., Jewitt, D. C., \& Fernández, Y. R. 2004, ApJ, 127, 2997

Hsieh, H. H., Jewitt, D., \& Fernández, Y. R. 2009, ApJ Lett., 694, L111

Hsieh, H. H., Jewitt, D., \& Ishiguro, M. 2009, ApJ, 137, 157

Jewitt, D., Chizmadia, L., Grimm, R., \& Prialnik, D., 2007, in: B. Reipurth, D. Jewitt \& K. Keil (eds.), Protostars and Planets $V$ (Tucson: Univ. Arizona Press), p. 863

Jewitt, D., Lacerda, P., \& Peixinho, N. 2007, IAUC 8847, 1

Jewitt, D., Yang, B., \& Haghighipour, N. 2009, ApJ, 137, 4313

Levison, H. F., Bottke, W. F., Nesvorný, D., Morbidelli, A., \& Gounelle, M. 2008, LPI 1405, 8156

McKinnon W. B., Prialnik, D., Stern, A. S., \& Coradini, A. 2007, in: M. A. Barucci, H. Boehnhardt, D. Cruikshank \& A. Morbidelli (eds.) The Solar System beyond Neptune (Tucson: Univ. Arizona Press), p. 213

Notesco, G. \& Bar-Nun, A. 1997, Icarus, 126, 336

Merk, R. \& Prialnik, D. 2006, Icarus, 183, 283

Podolak, M. \& Prialnik, D. 2006, in: P. J. Thomas, R. D. Hicks, C. F. Chyba \& C. P. McKay (eds.), Comets and the Origin and Evolution of Life, (Berlin: Spriger), p. 303

Prialnik, D. 1992, ApJ, 388, 196

Prialnik, D., Benkhoff, J., \& Podolak, M. 2004, in: M. C. Festou, H. U. Keller, H. A. Weaver \& M. Festou (eds.), Comets II, (Tucson: Univ. Arizona Press), p. 359

Prialnik, D. \& Merk, R. 2008, Icarus, 197, 211

Prialnik, D. \& Rosenberg, E. D. 2009, MNRAS, 399, L79

Sarid, G., Prialnik, D., Meech, K. J., Pittichovà, J., \& Farnham, T. L. 2005, PASP, 117, 796

Schorghofer, N. 2008, ApJ, 682, 697

Toth, I. 2000, $A \mathscr{E} A, 360,375$ 